\section{Smoking is associated with early nodular disease in RF-positive RA}

Researchers in Sweden have confirmed that ever having smoked is a strong risk factor for the early development of rheumatoid nodules. Their nested, case-control study found that rheumatoid factor (RF)-positive current or former smokers with rheumatoid arthritis (RA) were much more likely to have early nodular disease-a marker of disease severity — compared with patients who had never smoked. No dosedependent effect of smoking on nodular disease was found, however, which might indicate that smoking has a greater effect on the initiation of nodular disease than on its progression.

Nyhäll-Wåhlin and colleagues recruited 336 patients with newly diagnosed RA, identified from the BARFOT (Better Antirheumatic Pharmacotherapy) register. All had been symptomatic for $\leq 1$ year and had never received disease-modifying antirheumatic drugs, which are known to increase the risk of developing rheumatoid nodules. The 112 case patients had nodular disease at inclusion on the register; the 224 control patients were matched for age, sex and disease duration. Patients' smoking status was recorded in the register; the authors also obtained a more detailed smoking history from 64 case patients and 146 controls, via a questionnaire.

The authors observed that there were more current smokers in the study cohort (33\%) than in the general Swedish population (20\%). Patients who smoked were more likely to be RFpositive than nonsmokers, although the association of smoking with early nodular disease persisted after controlling for RF. The authors speculate that RF might mediate the effects of smoking on RA.

Original article Nyhäll-Wåhlin B-M et al. (2006) Smoking is a strong risk factor for rheumatoid nodules in early rheumatoid arthritis. Ann Rheum Dis 65: 601-606

\section{TNF inhibitors plus cyclophosphamide could increase patients' cancer risk}

Tumor necrosis factor (TNF) induces apoptosis in several tumor types. TNF inhibitors have become a mainstay of treatment in many inflammatory conditions, although it has been suggested that these agents could increase patients' risk of developing cancer. To date, these concerns have principally focused on lymphoma; however, Stone et al. now report that patients treated with both a TNF inhibitor and cyclophosphamide might have an increased risk of developing solid tumors, beyond the risk attributable to cyclophosphamide alone.

The authors had previously reported that solid tumors developed in six patients assigned to the etanercept arm of the Wegeners' Granulomatosis Etanercept Trial (WGET), in which 180 patients with active Wegener's granumolatosis were randomly allocated to receive either etanercept or placebo, in addition to standard care. All six patients with tumors had also received cyclophosphamide treatment. There were no malignancies among patients in the placebo arm during the trial (median follow-up 27 months).

Subsequently, the authors calculated that 1.92 malignancies would have been expected to occur in the etanercept arm. During the 3-month observation period after the trial ended, a further three patients developed malignancies, two of whom had been treated with a TNF inhibitor. Overall, 8 of 9 patients who developed a tumor had been treated with a TNF inhibitor and cyclophosphamide.

These findings emphasize the importance of long-term follow-up of patients who are treated with TNF inhibitors, say the authors. They speculate that tumors associated with TNF inhibition might develop more rapidly in patients who also receive immunosuppressive therapy.

Original article Stone JH et al. (2006) Solid malignancies among patients in the Wegener's granulomatosis etanercept trial. Arthritis Rheum 54: 1608-1618

\section{Lymphoma risk is elevated even in new-onset inflammatory polyarthritis}

Lymphoma is more than twice as likely to occur in patients with inflammatory polyarthritis than in the general population, a UK study has shown-supporting previous findings of a causal link between the incidence of inflammatory polyarthritis (which includes rheumatoid arthritis $[R A])$ and lymphoma. Whether lymphoma develops as a consequence of immunosuppressive treatment, or of the characteristics of RA or 